\title{
The Spitzer search for the transits of HARPS low-mass planets
}

\section{No transit for the super-Earth HD $40307 \mathrm{~b}$}

\author{
M. Gillon ${ }^{1,2}$, D. Deming ${ }^{3}$, B.-O. Demory ${ }^{4,2}$, C. Lovis $^{2}$, S. Seager ${ }^{4}$, M. Mayor ${ }^{2}$, F. Pepe ${ }^{2}$, D. Queloz ${ }^{2}$, D. Segransan ${ }^{2}$, \\ S. Udry ${ }^{2}$, S. Delmelle ${ }^{1}$, and P. Magain ${ }^{1}$
}

1 Institut d'Astrophysique et de Géophysique, Université de Liège, Allée du 6 Août 17, Bat. B5C, 4000 Liège, Belgium e-mail: michael.gillon@ulg.ac.be

2 Observatoire de Genève, Université de Genève, 51 Chemin des Maillettes, 1290 Sauverny, Switzerland

3 Planetary Systems Branch, Code 693, NASA/Goddard Space Flight Center, MD 20771 Greenbelt, USA

4 Department of Earth, Atmospheric and Planetary Sciences, Department of Physics, Massachusetts Institute of Technology, 77 Massachusetts Ave., MA 02139 Cambridge, USA

Received 27 January 2010 / Accepted 14 April 2010

ABSTRACT

\begin{abstract}
We used Spitzer and its IRAC camera to search for the transit of the super-Earth HD 40307b. The hypothesis that the planet transits could not be firmly discarded from our first photometric monitoring of a transit window because of the uncertainty coming from the modeling of the photometric baseline. To obtain a firm result, two more transit windows were observed and a global Bayesian analysis of the three IRAC time series and the HARPS radial velocities was performed. Unfortunately, the hypothesis that the planet transited during the observed phase window is firmly rejected, while the probability that the planet does transit but that the eclipse was missed by our observations is nearly negligible $(0.26 \%)$.
\end{abstract}

Key words. binaries: eclipsing - planetary systems - stars: individual: HD 40307 - techniques: photometric

\section{Introduction}

Transiting extrasolar planets are and will remain key objects for our study and understanding of the large planetary population hosted by our galaxy. Except for the planets of our own solar system, transiting exoplanets are the only ones we can accurately estimate for mass, radius, and, by inference, constraints on internal composition. Furthermore, their special geometrical configuration gives us the opportunity to study their atmospheres directly, without the challenging need to spatially resolve their light from that of their host star. Occultation photometry and spectroscopy has been used to map the thermal emission of more than a dozen highly irradiated giant planets and to assess their atmospheric compositions, vertical thermal gradients, and albedos (see, e.g., Deming 2009). More demanding in terms of signal-to-noise ratio (SNR), transit transmission spectroscopy has been used to detect atomic and molecular features in the atmospheres of the two giant planets HD 209458b and HD 189733b (e.g., Charbonneau et al. 2002; Vidal-Madjar et al. 2003; Swain et al. 2009). These transit and occultation measurements have opened a new field in astronomy: exoplanetary science.

One of the next major steps in this field will be the first atmospheric characterization of a terrestrial extrasolar planet. Radial velocity (RV) surveys have opened the road to this goal by revealing a population of planets of a few Earth masses (e.g., Udry et al. 2007; Mayor et al. 2009a,b), the so-called "super-Earths". Last year, the CoRoT space mission detected the first of these

^ The photometric time series used in this work are only available in electronic form at the CDS via anonymous ftp to cdsarc.u-strasbg.fr (130.79.128.5) or via http://cdsweb.u-strasbg.fr/cgi-bin/qcat?J/A+A/518/A25
super-Earths that transits its parent star: CoRoT-7b (Léger et al. 2009; Queloz et al. 2009). With a mass $M_{\mathrm{p}}=4.8 \pm 0.8 M_{\oplus}$ and a radius $R_{\mathrm{p}}=1.7 \pm 0.1 R_{\oplus}$, CoRoT-7b has an average density of $5.6 \pm 1.3 \mathrm{~g} \mathrm{~cm}^{-3}$, similar to the Earth's. Still, its structure is quite uncertain, because its measured mass and radius are consistent with both an Earth-like and a volatile-rich composition (Valencia et al. 2009). Due to the relative faintness of the host star $(V=11.7, K=9.8)$ and the small planet-star size ratio $\left(3.4 \times 10^{-4}\right)$, a thorough characterization of this planet is unfortunately not possible with existing or planned instruments.

The detection of a second transiting super-Earth was recently announced by the MEarth Project (Charbonneau et al. 2009). This new planet orbits around the nearby M-dwarf GJ 1214 (M4.5V, $d=13 \mathrm{pc}$ ). Its mass is $M_{\mathrm{p}}=6.5 \pm 1 M_{\oplus}$, similar to the one of CoRoT-7b. Nevertheless, GJ 1214b has a much larger radius than CoRoT-7b: $R_{\mathrm{p}}=2.68 \pm 0.13 R_{\oplus}$. The resulting average density of $1.9 \pm 0.4 \mathrm{~g} \mathrm{~cm}^{-3}$ suggests the presence of a significant gas component. GJ 1214 b is thus more a kind of "mini-Neptune" than a massive rocky planet, but its actual composition remains uncertain, as shown by Rogers \& Seager (2009). GJ 1214b is a favorable target for atmospheric measurements able to provide valuable insight into its composition and history. In particular, the planned James Webb Space Telescope (Gardner et al. 2006) will have the potential to thoroughly characterize the atmosphere of this super-Earth, mainly because of the infrared brightness ( $K=8.8)$ and small size $\left(0.21 R_{\odot}\right)$ of its host star. Its transit depth $(1.4 \%)$ is indeed virtually the same as that of one of the best-studied hot Jupiters, HD 209458 b (1.5\%).

The discovery of GJ $1214 \mathrm{~b}$ illustrates the critical role that low-mass planets transiting nearby bright stars can play in enabling progress in this area. Due to their need to observe 
thousands of stars at once, most transit surveys do not target the brightest and nearest stars. On the contrary, Doppler surveys target a large number of very bright stars. In this context, it is not surprising that the two best studied hot Jupiters, HD 209458b and HD 189733b, were first detected by RV measurements and caught afterwards in transit (Charbonneau et al. 2000; Henry et al. 2000; Bouchy et al. 2005). This was also the case for the hot Neptune GJ 436b (Butler et al. 2004; Gillon et al. 2007b), also an excellent target for detailed follow-up atmospheric studies (see, e.g., Deming et al. 2007; Demory et al. 2007). Searching for the transits of the low-mass planets detected by RV measurements is thus an obvious method of detecting transiting super-Earths suitable for a thorough atmospheric characterization. Doppler surveys have now detected enough nearby low-mass planets to make it highly probable that at least some of them transit their parent stars. In particular, our HARPS Doppler survey (Mayor et al. 2003) has now detected more than 40 low-mass planets (Lovis et al. 2009). Among them are several announced hot Neptunes (see, e.g., Santos et al. 2004; Bouchy et al. 2009) and super-Earths (see, e.g., Udry et al. 2007; Forveille et al. 2008), but also many more firmly confirmed planets that are waiting for a final analysis or additional RV measurements to fully characterize their orbits.

Differential photometric precisions of $0.1 \%$ are now routinely achieved from the ground, making it straightforward to detect the transit of a hot Jupiter. Due to the small size of its host star, the transit of the hot Neptune GJ 436b could also be detected from the ground (Gillon et al. 2007b). For most of the low-mass planets detected by HARPS around solar-type stars, a transit detection requires a much more challenging photometric precision, $\sim 50 \mathrm{ppm}$ or better on a time scale of a few hours (corresponding to the mean transit duration for a close-in planet), and for very bright stars. From the ground, such a precision is presently out of reach. While the photon noise would not be a problem even for relatively small telescopes, any atmospheric instability creates a correlated noise with a magnitude larger than the one cited above. Differential photometry using multiple stars within the field of view to remove atmospheric and instrument effects helps to reduce the amplitude of this correlated noise, and ground-based sub-mmag transit light curves nearly free of correlated noise have been obtained for a few transiting planets (see, e.g., Gillon et al. 2009; Johnson et al. 2009; Winn et al. 2009). These observations were made for relatively faint stars $(V>10)$ with several comparison stars of similar brightness nearby (at a few arcmin at most), making it possible to obtain an excellent correction of atmospheric effects. Most bright nearby stars do not have suitably bright comparison stars that fall within the same field of view. Furthermore, the transit ephemeris of the very low-mass planets detected by HARPS generally have a 2- $\sigma$ probability interval of a dozen hours or more. This is not only because of the low amplitude of the RV signal due to the planet, but also because of the RV low-frequency noise of the host star and the tendency of low-mass planets to be found in multiple-planet systems (Lovis et al. 2009). Due to the day-night cycle and to the fact that only a fraction of some nights is suitable for precise photometry of the target, ground-based telescopes are definitely not the best option for such a transit search, and a space-based instrument is required.

The needed space telescope has to be able to perform exquisitely precise photometry for bright stars but also to monitor the same stars continuously for dozens of hours at a time. We have concluded that the best choice for such a program would be the Spitzer Space Telescope (Werner et al. 2004). Due to its heliocentric orbit, it can monitor most of the stars for several weeks during their visibility windows, but it has also demonstrated on many instances its excellent photometric potential, detecting signals with an amplitude a few hundred of ppm (see, e.g., Beaulieu et al. 2007). We have thus set up a Spitzer program devoted to the search for transits of HARPS low-mass planets. This program is presently divided in two Spitzer sub-programs: a cycle 5 DDT program (495) devoted to only the planet HD 40307b (Mayor et al. 2009a, hereafter M09), and a 100 hour cycle 6 DDT program (60027) devoted to $\sim 10$ other low-mass planets.

This paper presents our results for HD 40307b. This superEarth $\left(M_{\mathrm{p}} \sin i=4.2 M_{\oplus}\right)$ orbits around a bright $(V=7.2, K=$ 4.8) nearby (12.8 pc) K2-dwarf. Because of its proximity to its host star $(a=0.047 \mathrm{AU})$, its geometric transit probability is $\sim 7 \%$, making it a good candidate for a transit search. Section 2 presents the data used in this work, including the Spitzer observations and their reduction. Our data analysis is presented in Sect. 3, and our results are presented and discussed in Sect. 4. We give our conclusions in Sect. 5 .

\section{Data}

\subsection{Radial velocities}

We used the 135 HARPS measurements presented in Mayor et al. (2009a) to estimate the HD 40307b transit windows for our Spitzer observations. We also used these data in our final global analysis aiming to assess the transit probability of the planet (see Sect. 3).

\subsection{Spitzer IRAC photometry}

HD 40307b was first observed with Spitzer's Infra-Red Array Camera (IRAC, Fazio et al. 2004) on 31 October 2008 from $01 \mathrm{~h}$ $02 \mathrm{UT}$ to $09 \mathrm{~h} 40 \mathrm{UT}$, corresponding to a $\sim 2-\sigma$ transit window of the planet as predicted by our RVs. With magnitude $K=4.8$ and K2V spectral type, HD 40307 is a very high signal-to-noise ratio (SNR) target for all IRAC bands. Considering the substantial experience of our team with Spitzer photometry at $8 \mu \mathrm{m}$ (e.g., Deming et al. 2007; Gillon et al. 2007a; Demory et al. 2007), we conservatively decided to observe in this channel (SiAs detector) and to use the established technique of continuous staring in non-dithered subarray mode. We choose to use an exposure time of $0.32 \mathrm{~s}$, the largest one for which the star would not be saturated on the detector. The Spitzer flux density estimator tool ${ }^{1}$ predicts a flux density of $806 \mathrm{mJy}$ for HD $40307 \mathrm{~b}$ at $8 \mu \mathrm{m}$, which translates into a SNR of $\sim 5100$ for a 1 minute integration when taking into account the photon, read-out and background noises, and the instrumental throughput corrections ${ }^{2}$.

Values for the stellar and planet sizes are needed to estimate the SNR that should be expected for the detection of a putative transit. Using $T_{\text {eff }}=4977 \pm 59 \mathrm{~K}, \log g=4.47 \pm 0.16$ and $[\mathrm{Fe} / \mathrm{H}]=-0.31 \pm 0.03(\mathrm{M} 09)$, the theoretical stellar calibration obtained by Torres et al. (2009) from well-constrained detached binary systems predicts a stellar mass of $0.78 \pm 0.06 M_{\odot}$, in good agreement with the estimation of Sousa et al. (2008), $0.77 \pm 0.05 M_{\odot}$. The parallax of this nearby star was precisely determined by Hipparcos (ESA 1200) to $\pi=77.95 \pm 0.53$ mas. From this value, the Hipparcos $V$ magnitude $(V=7.17$, ESA $1200)$, the 2MASS $K$ magnitude $(K=4.79$, Skrutskie et al. 2006), the spectroscopic parameters from M09 and the bolometric correction calibration presented by Masana et al. (2006), we

\footnotetext{
1 http://ssc.spitzer.caltech.edu/tools/starpet/

2 http://ssc.spitzer.caltech.edu/documents/som/
} 


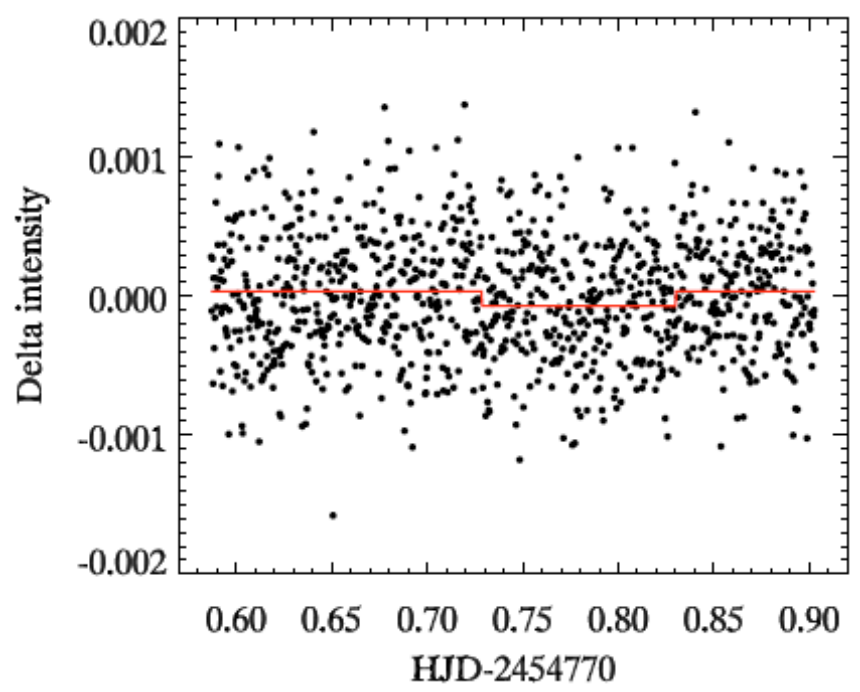

Fig. 1. HD 40307 Spitzer $8 \mu \mathrm{m}$ photometry (first run) obtained after reduction by DD. The first $3 \mathrm{~h}$ have been cut off and a line was fitted in the photometry to correct for the "ramp". The best-fitting central transit model is overimposed. See text for details.

deduce a radius of $0.68 \pm 0.02 R_{\odot}$ for the star (corresponding to a luminosity $\left.L_{*}=0.26 \pm 0.01 L_{\odot}\right)$. Depending on the unknown planetary composition, the radius of HD $40307 \mathrm{~b}$ could lie between 1.1 and $5 R_{\oplus}$ (Seager et al. 2007), with the lowest value corresponding to an unlikely pure iron composition. Assuming $R_{*}=0.68 \pm 0.02 R_{\odot}, R_{\mathrm{p}}=1.1 R_{\oplus}$ translates into a transit depth of $220 \pm 13 \mathrm{ppm}$ and a maximum transit duration of $\sim 135 \mathrm{~min}$. Assuming an actual duration of $30 \mathrm{~min}$ (and thus a large impact parameter), the resulting expected SNR on the transit detection is $\sim 6$. Thus we should have been able to detect the transit of the planet for any plausible composition and for most of the possible impact parameters.

The data of this run consist of 1297 sets of 64 individual subarray images. Two of us (DD and MG) performed an independent preliminary reduction of these images. Both of us used aperture photometry on the Basic Calibrated Data (BCD) provided by the standard Spitzer reduction pipeline. DD used his own aperture photometry algorithm with an aperture of 3 pixels. On his side, MG used the IRAF/DAOPHOT ${ }^{3}$ software (Stetson 1987), and obtained the best results with a relatively large aperture of 6 pixels encompassing most of the wings of the bright star's point-spread function (PSF). After a rejection of the discrepant fluxes, the measurements were averaged for each set of 64 images, leading thus to 1297 measurements in the final light curves. The systematic effect known as the "ramp" (see, e.g., Knutson et al. 2007) is extremely sharp at the beginning of the run (see Fig. 3). In his preliminary analysis, DD simply cut off the first three hours and fitted a straight line to the remainder. Figure 1 shows the residuals of this fit vs. HJD. A tiny drop in brightness is visible at $\sim 2454770.70$ HJD. Some tests were done to assess its significance. A transit curve having a fixed duration - calculated for a central transit - was constructed and fitted as a function of center time and transit depth. The best fit is overplotted in Fig. 1. From the precision on its depth and on the out-of-transit level, its significance is $\sim 3 \sigma$. It is thus a marginal detection. 50000 bootstrap trials were done wherein data were

3 IRAF is distributed by the National Optical Astronomy Observatory, which is operated by the Association of Universities for Research in Astronomy, Inc., under cooperative agreement with the National Science Foundation.

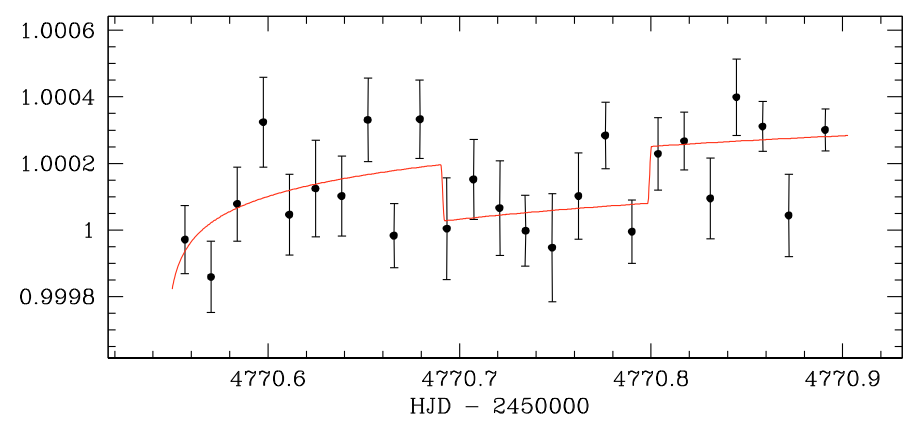

Fig. 2. HD 40307 Spitzer $8 \mu$ m photometry obtained after reduction by MG. The first hour has been cut off and the points have been binned per $20 \mathrm{~min}$. The best-fitting ramp+transit model is overimposed. See text for details.

permuted and the maximum amplitude of each set was found, varying the center time. Only 1-percent of the trials produced an amplitude larger than the one of the structure detected. Varying the aperture over the range 2.5 to 5 pixels, this fraction ranged from zero to $2 \%$, so a $98 \%$ confidence could be assigned to the transit-like structure under the simple line model for the baseline.

MG cut off the first hour of data and obtained a light curve that shows a similar drop in brightness (see Fig. 2). Its significance was tested with a Markov Chain Monte Carlo method (see Sect. 3). Two Markov chains were performed. For each of them, the free parameters were the transit depth, the impact parameter, the central timing and the parameters of the baseline model (see Sect. 3 for details). The stellar mass and radius were fixed to $M_{*}=0.78 \pm 0.06 M_{\odot}$ and $R_{*}=0.68 \pm 0.02 R_{\odot}$. For the first chain, the initial transit depth was set to $300 \mathrm{ppm}$, while it was set to zero for the second chain. The results for both chains were analyzed together to obtain the posterior distribution for all fitted parameters. The solutions for both chains are fully compatible, the deduced transit depth being $\mathrm{d} F=170_{-62}^{+49} \mathrm{ppm}$. The significance based on this analysis is thus comparable to the one deduced from the analysis performed by DD. The deduced transit depth translates into a planet radius $R_{\mathrm{p}}=0.97_{-0.2}^{+0.13} R_{\oplus}$. Such a small radius could be estimated a priori unlikely, because it corresponds to a planet denser than expected for a pure iron composition. Still, any unknown systematic error in the measurements used to estimate the stellar radius could lead to a larger planet. For instance, the stellar calibration of Torres et al. (2009) leads to a larger stellar radius, $0.86 \pm 0.17 R_{\odot}$. We thus chose not to discard our marginal transit detection on the basis that it was shallower than expected, and we tried to confirm it with more observations.

We requested the observation of two more transit windows with Spitzer. HD 40307 was again observed with IRAC on 02 February 2009 from 21 h 26 to 05 h 52 UT and on 13 March 2009 from 16 h 23 to 00 h 48 UT. For both runs, 1108 set of 64 individual subarray images were acquired at $8 \mu \mathrm{m}$, again with an exposure time of $0.32 \mathrm{~s}$. At this stage, it was decided to obtain a final light curve for each run and to perform a global Bayesian analysis of these light curves in addition to the HARPS measurements to assess the probability that HD 40307b was transiting (see Sect. 3). For the three Spitzer runs, we converted fluxes from the Spitzer units of specific intensity (MJy/sr) to photon counts, and aperture photometry was obtained for HD 40307 in each image using IRAF/DAOPHOT. A circular aperture with a radius of 6 pixels was centered in each image by fitting a Gaussian profile on the target. A mean sky background was measured in 


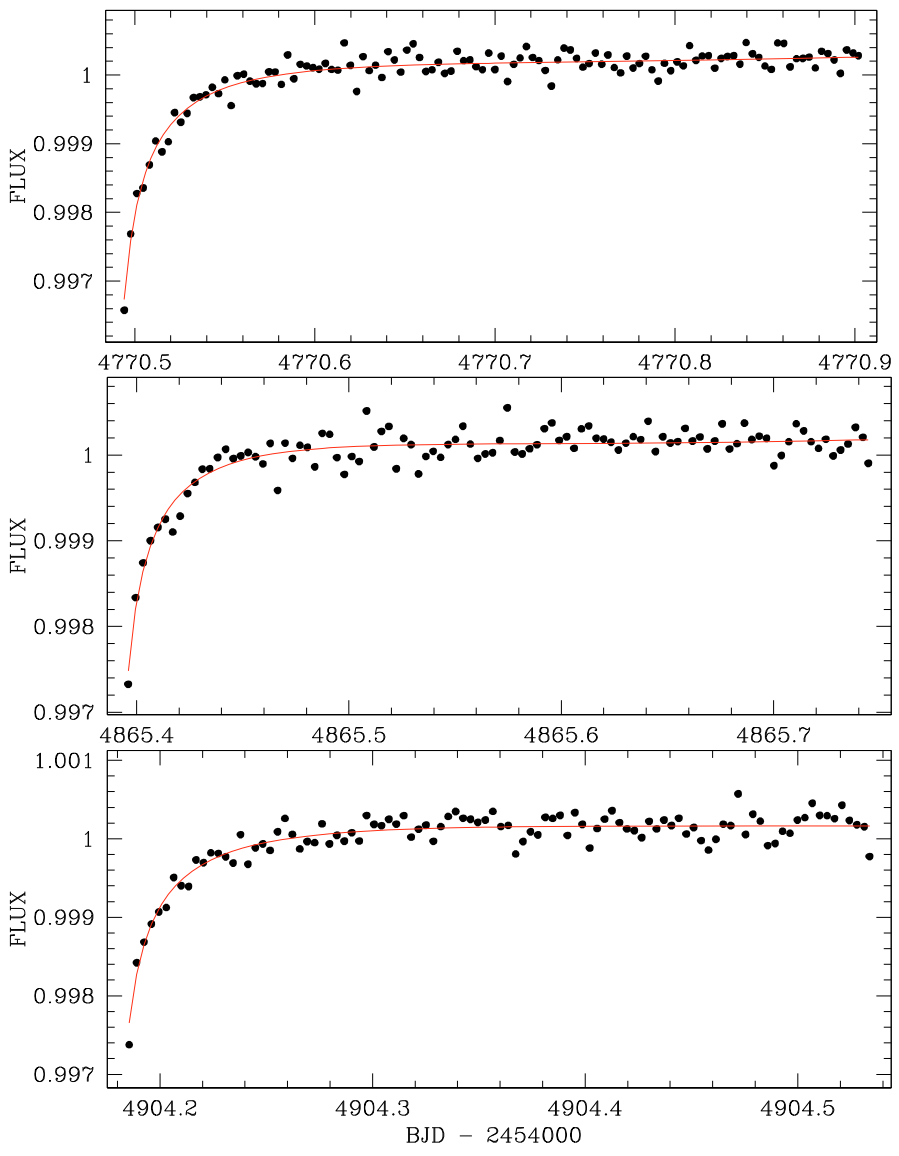

Fig. 3. HD 40307 Spitzer $8 \mu \mathrm{m}$ photometry binned with an interval of five minutes. The best-fitting model is overimposed. See text for details.

an annulus extending from 16 to 24 pixels from the center of the aperture, and subtracted from the measured flux for each image. We discarded the possibility of contamination in our background estimates from the wings of the star's PSF by trying different background annuli that gave similar results. For each set of 64images, a 3- $\sigma$ median clipping was used to reject outliers, then the remaining values were averaged and the error on the mean was considered as the error on the resulting measurement. The mean number of measurements rejected per set for the three runs together was 1.5. For all the sets, the first subarray image showed systematically lower fluxes than the others. For the two other Spitzer runs, the last subarray measurement was also systematically rejected because it was significantly larger than the others. At this stage, a moving median filtering was used to reject outlier sets. It used a 4- $\sigma$ clipping and a window function of 20 measurements. For the first run, two measurements were rejected out of 1297 . For both the second and third run, three measurements were rejected out of 1108 . The resulting light curves binned with an interval of five minutes are shown in Fig. 3.

\section{Data analysis}

The three Spitzer $8 \mu \mathrm{m}$ time series were first binned with an interval of five minutes to speed up the analysis, a sampling of $5 \mathrm{~min}$ being good enough for the proper modeling of the relatively smooth photometric baselines and for the possible detection of the expected transit (except for an unlikely very grazing transit). These binned photometric time series and the HARPS measurements were used as input data in a global analysis aiming to determine the probability that HD $40307 \mathrm{~b}$ transits and, if so, its transit and physical parameters. The analysis was performed with the adaptative Markov Chain Monte Carlo (MCMC) algorithm presented in Gillon et al. (2010). The assumed models were based on a star and three planets on a Keplerian orbit about their common center of mass (the planet-planet interactions were first assessed and revealed to be negligible). The planet HD 40307b had its orbital inclination free and was thus allowed to transit the star. As no transit of the planets $\mathrm{c}$ and $\mathrm{d}$ was expected to happen during our Spitzer runs, we fixed their inclination to 90 degrees. To model the eclipse photometry, we used the photometric eclipse model of Mandel \& Agol (2002) multiplied by a systematic effect model. For the RVs, a classical Keplerian model was used in addition to a linear drift (see M09) and a Rossiter-McLaughlin (RM) effect model (Giménez 2006) for the RVs obtained during a transit.

Considering the very red bandpass of our Spitzer photometry, we assumed no limb-darkening for the photometry. For the RVs, a quadratic limb-darkening was assumed. The values $u_{1}=0.613$ and $u_{2}=0.161$ were deduced from Clarets tables $(2000 ; 2004)$ for the stellar parameters presented in M09 and for the $V$-filter, corresponding to the maximum transmission of HARPS. These values were kept fixed in the MCMC.

The $8 \mu \mathrm{m}$ IRAC photometry is known to be affected by a systematic effect causing the gain to increase asymptotically over time for every pixel, with an amplitude depending on their illumination history (see e.g. Knutson et al. 2008 and references therein). Following Charbonneau et al. (2008), this "ramp" was modelled in each IRAC time series as a quadratic function of $\ln (\mathrm{d} t)$ :

$$
A(\mathrm{~d} t)=a_{1}+a_{2} \ln (\mathrm{d} t)+a_{3}(\ln (\mathrm{d} t))^{2}+a_{4} \mathrm{~d} t,
$$

where $\mathrm{d} t$ is the elapsed time since $15 \mathrm{~min}$ before the start of the run. The linear term $a_{4} \mathrm{~d} t$ was added to take into account that HD 40307 is a slowly rotating K-dwarf star that could be variable on a time scale of several weeks, and that such a lowfrequency variability could translate into a slight slope in the Spitzer time series. Furthermore, we compared the results obtained when ramp models with and without the $a_{4} \mathrm{~d} t$ term are fitted by least-square minimization on the three IRAC time-series. We used the BIC criterion (e.g., Carlin \& Louis 2008) to perform this comparison, and the results were that the model with the a4 term is $\sim 5220$ times more likely for the first IRAC time-series, $\sim 660$ times more likely for the second time-series, and $\sim 2$ times less likely for the third time-series. The model with the linear term $a_{4} \mathrm{~d} t$ appears thus to be a better representation of the variation of the measured flux caused by the stellar and instrumental sensitivity variability.

The photometric correlated noise and the RV jitter noise were taken into account as in Gillon et al. (2010). For the three planets orbiting HD 40307, the jump parameters in the analysis were the two Lagrangian parameters $e \cos \omega$ and $e \sin \omega$ where $e$ is the orbital eccentricity and $\omega$ is the argument of periastron, the orbital period $P$, the $K_{2}$ parameter characterizing the amplitude of the orbital RV signal (see Gillon et al. 2010) and the time $T_{0}$ for which the true anomaly $M=\pi / 2-\omega$. For a transiting planet, $T_{0}$ represents the time of minimum light. For HD $40307 \mathrm{~b}$, the planet/star area ratio $\left(R_{\mathrm{p}} / R_{\mathrm{s}}\right)^{2}$ and the impact parameter $b^{\prime}=a \cos i / R_{*}$ were also jump parameters.

Because of the expected small planet-star area ratio and the low rotational velocity of the $\operatorname{star}\left(V \sin I<1 \mathrm{~km} \mathrm{~s}^{-1}, \mathrm{M} 09\right)$, the amplitude of the RM anomaly for a transit of HD 40307b would be smaller than the typical precision of the HARPS measurements, so we fixed the projected rotational spin velocity of the star $V \sin I$ to $1 \mathrm{~km} \mathrm{~s}^{-1}$ and the projected spin-orbit angle $\beta$ of 
Table 1. Median and 1- $\sigma$ limits of our posterior distributions for the parameters of the three planets orbiting around HD 40307.

\begin{tabular}{lcccc}
\hline \hline Parameter & HD 40307b & HD 40307c & HD 40307d & Units \\
\hline Jump parameters & & & & \\
\hline Transit epoch $T_{0}-2450000$ & $4770.78 \pm 0.11$ & $4957.73 \pm 0.26$ & $4946.02_{-0.51}^{+0.50}$ & HJD \\
Orbital period $P$ & $4.31109_{-0.00052}^{+0.00051}$ & $9.6183 \pm 0.0024$ & $20.4434_{-0.0092}^{+0.0095}$ & days \\
$e \cos \omega$ & $0.009 \pm 0.060$ & $0.031_{-0.048}^{+0.049}$ & $0.016_{-0.049}^{+0.050}$ & \\
$e \sin \omega$ & $0.038_{-0.059}^{+0.057}$ & $0.027_{-0.049}^{+0.050}$ & $0.048 \pm 0.042$ & \\
RV $K_{2}$ & $3.22 \pm 0.19$ & $5.09 \pm 0.25$ & $7.05_{-0.33}^{+0.34}$ & \\
Planet/star area ratio $\left(R_{\mathrm{p}} / R_{\mathrm{s}}\right)^{2}$ & $0.0022_{-0.0011}^{+0.0015}$ & - & - & \\
$b^{\prime}=a \cos i / R_{*}$ & $7.3_{-4.3}^{+4.7}$ & - & - & $R_{*}$ \\
\hline Deduced parameters & & & & \\
\hline RV $K$ & $1.99 \pm 0.12$ & $2.40 \pm 0.12$ & $2.59 \pm 0.12$ & $\mathrm{~m} \mathrm{~s}{ }^{-1}$ \\
Orbital semi-major axis $a$ & $0.0477_{-0.0013}^{+0.0012}$ & $0.0815_{-0.0021}^{+0.0020}$ & $0.1348_{-0.0035}^{+0.0033}$ & $\mathrm{AU}$ \\
Orbital eccentricity $e$ & $0.077_{-0.038}^{+0.047}$ & $0.068_{-0.034}^{+0.022}$ & $0.072_{-0.034}^{+0.040}$ & \\
Argument of periastron $\omega$ & $78_{-73}^{+82}$ & $37_{-74}^{+60}$ & $72_{-50}^{+57}$ & $\mathrm{deg}$ \\
$M_{\mathrm{p}} \sin i$ & $4.27_{-0.33}^{+0.35}$ & $6.80_{-0.52}^{+049}$ & $9.28_{-0.63}^{+0.69}$ & $M_{\oplus}$ \\
Orbital inclination $i$ & $61_{-24}^{+17}$ & - & - & $\mathrm{deg}$ \\
\hline
\end{tabular}

Notes. Jump parameters are the model parameters that are randomly perturbed at each step of the MCMC.

HD $40307 \mathrm{~b}$ to $0 \mathrm{deg}$. It is also worth noticing that because of the tiny amplitude of the RM anomaly expected for HD 40307b, the HARPS data alone could not provide any meaningful constraints on the possible transiting nature of the planet.

A value for the stellar mass and radius were drawn at each step of the Markov chains from the distributions $N\left(0.78,0.06^{2}\right) M_{\odot}$ and $N\left(0.68,0.02^{2}\right) R_{\odot}$. A uniform prior distribution was assumed for each jump parameter. For each Spitzer time series, the four parameters of the baseline model were determined at each step of the MCMC by least-square minimization (see Gillon et al. 2010, for details). This was also the case for the systemic RV of the star. The planet radius was restricted to range from 1 to $6 R_{\oplus}$ to avoid unrealistic solutions.

The analysis was divided into two steps. A first Markov chain was performed to assess the level of correlated noise in the photometry and the jitter noise in the RVs and to update the measurement error bars accordindly. A value of $0.85 \mathrm{~m} \mathrm{~s}^{-1}$ was deduced for the RV jitter noise, while $\beta_{\text {red }}=[1,1,1.14]$ were deduced for the photometric time series (see Gillon et al. 2010 , for details). Five new chains $\left(10^{6}\right.$ steps each) were then performed using the updated measurement error bars. The good convergence and mixing of these five chains was checked succesfully using the Gelman \& Rubin (1992) statistic.

\section{Results and discussion}

Table 1 shows the median values and $68.3 \%$ intervals of the posterior distributions for the parameters of the three planets. These values are in good agreement with the ones reported by M09. Figure 3 shows the three IRAC time series with the best-fitting model overimposed. No transit is present for the best-fitting solution. The best-fitting RV models are shown with the RV measurements in Fig. 4.

Our global Bayesian analysis leads to a posterior transit probability for HD $40307 \mathrm{~b}$ of $0.3 \%$. If we consider only full transits, the posterior probability is only $0.19 \%$. The marginal transit detection that we obtained from the first IRAC run is thus unfortunately not confirmed, and the transiting nature of HD $40307 \mathrm{~b}$ is rejected at $\sim 3 \sigma$. As shown in Fig. 5, the fraction of the simulations for which HD $40307 \mathrm{~b}$ transits concern mostly

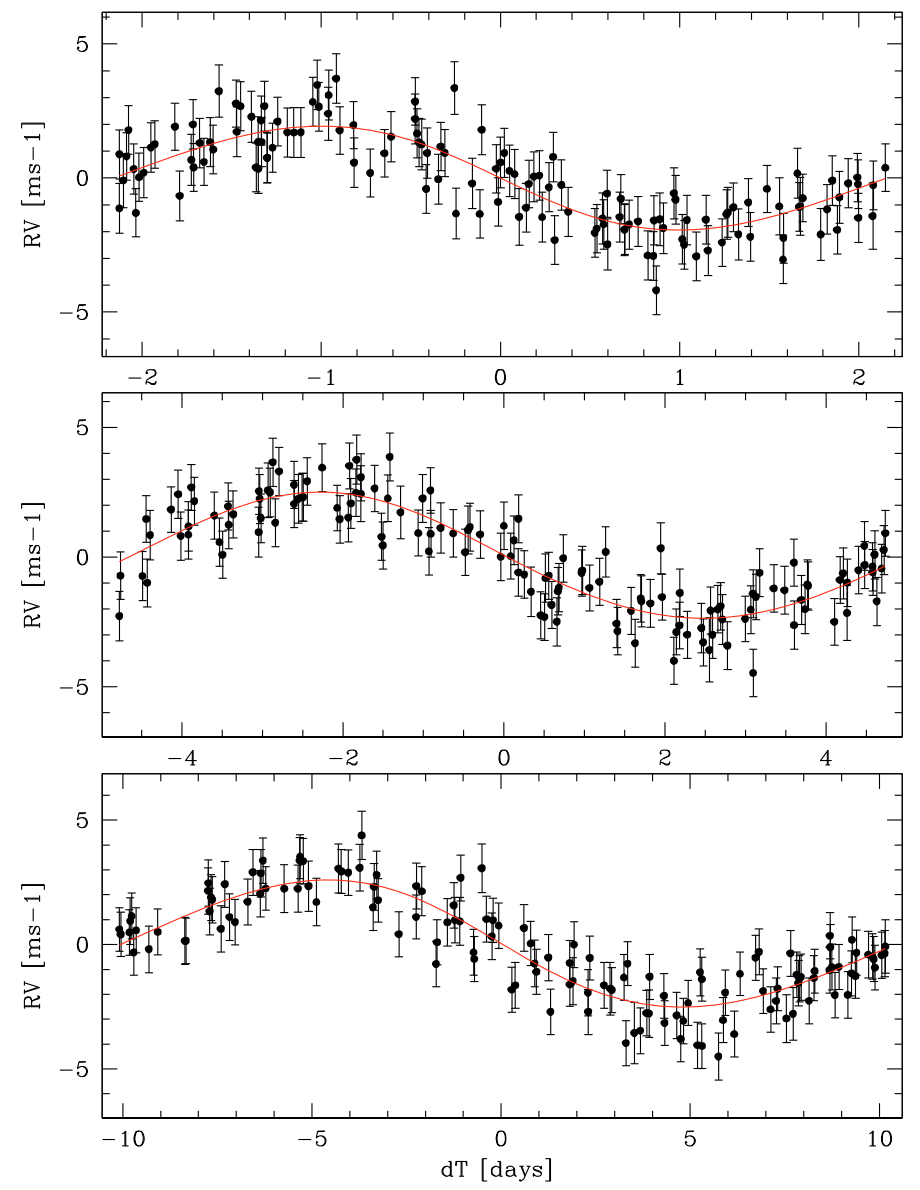

Fig. 4. Period-folded HARPS measurements and Keplerian curve for each of the planets, after correction of the effect of the two other planets and the drift. Top: HD 40307b, middle: HD 40307c, bottom: HD 40307d.

transits that happen outside the observed window. The resulting posterior probability that a transit was present in the observation window is $0.04 \%$, while no simulation resulted in a full transit 
Table 2. Median and 1- $\sigma$ limits of our posterior distributions for the parameters of the three planets orbiting around HD 40307.

\begin{tabular}{lcccc}
\hline \hline Parameter & HD 40307b & HD 40307c & HD 40307d & Units \\
\hline Jump parameters & & & & \\
\hline Transit epoch $T_{0}-2450000$ & $4770.78 \pm 0.11$ & $4957.73 \pm 0.26$ & $4946.02_{-0.51}^{+0.50}$ & HJD \\
Orbital period $P$ & $4.31109_{-0.00052}^{+0.00051}$ & $9.6183 \pm 0.0024$ & $20.4434_{-0.0092}^{+0.0095}$ & days \\
$e \cos \omega$ & $0.009 \pm 0.060$ & $0.031_{-0.048}^{+0.049}$ & $0.016_{-0.049}^{+0.050}$ & \\
$e \sin \omega$ & $0.038_{-0.059}^{+0.057}$ & $0.027_{-0.049}^{+0.050}$ & $0.048 \pm 0.042$ & \\
RV $K_{2}$ & $3.22 \pm 0.19$ & $5.09 \pm 0.25$ & $7.05_{-0.33}^{+0.34}$ & \\
Planet/star area ratio $\left(R_{\mathrm{p}} / R_{\mathrm{s}}\right)^{2}$ & $0.0022_{-0.0011}^{+0.0015}$ & - & - & \\
$b^{\prime}=a \cos i / R_{*}$ & $7.3_{-4.3}^{+4.7}$ & - & - & $R_{*}$ \\
\hline Deduced parameters & & & & \\
\hline RV $K$ & $1.99 \pm 0.12$ & $2.40 \pm 0.12$ & $2.59 \pm 0.12$ & $\mathrm{~m} \mathrm{~s}{ }^{-1}$ \\
Orbital semi-major axis $a$ & $0.0477_{-0.0013}^{+0.0012}$ & $0.0815_{-0.0021}^{+0.0020}$ & $0.1348_{-0.0035}^{+0.0033}$ & $\mathrm{AU}$ \\
Orbital eccentricity $e$ & $0.077_{-0.038}^{+0.047}$ & $0.068_{-0.034}^{+0.042}$ & $0.072_{-0.034}^{+0.040}$ & \\
Argument of periastron $\omega$ & $78_{-73}^{+82}$ & $37_{-74}^{+60}$ & $72_{-50}^{+57}$ & $\mathrm{deg}$ \\
$M_{\mathrm{p}} \sin i$ & $4.27_{-0.33}^{+0.35}$ & $6.80_{-0.52}^{+049}$ & $9.28_{-0.63}^{+0.69}$ & $M_{\oplus}$ \\
Orbital inclination $i$ & $61_{-24}^{+17}$ & - & - & $\mathrm{deg}$ \\
\hline
\end{tabular}

Notes. Jump parameters are the model parameters that are randomly perturbed at each step of the MCMC.

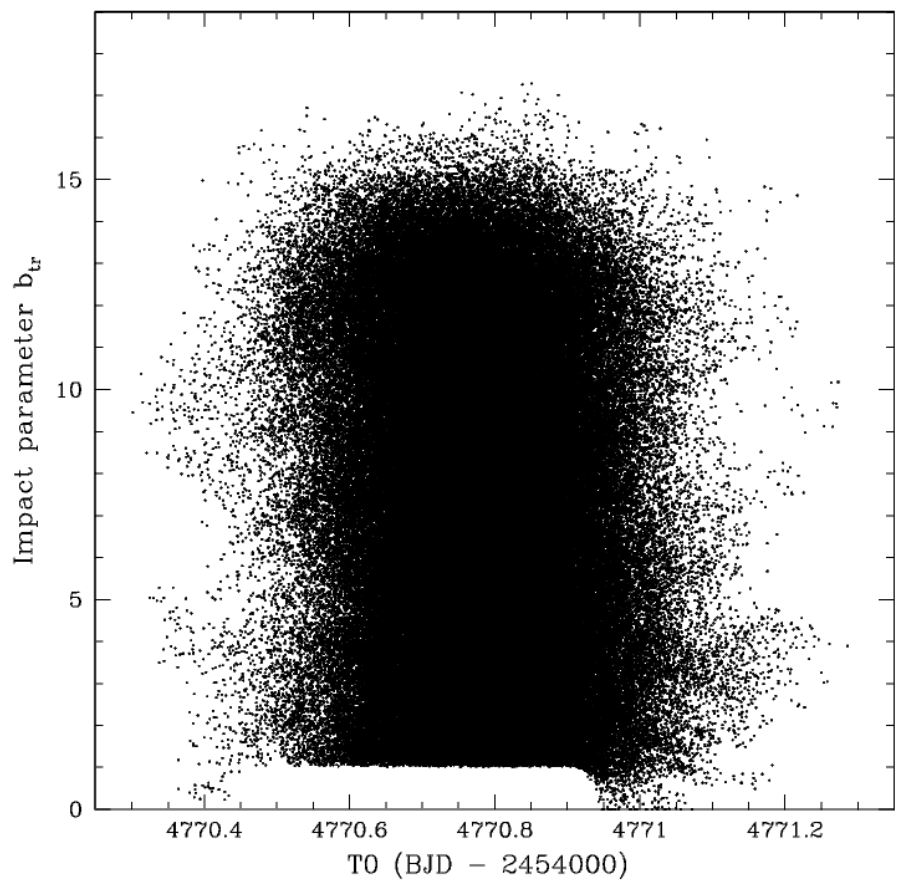

Fig. 5. Posterior probability density: time of minimum light $T_{0}$ vs. transit impact parameter $b_{\text {tr }}$. This bottom of this diagram shows clearly the parameter space cleared out by the Spitzer photometry.

present in the IRAC runs. The presence of a transit in the Spitzer data is thus rejected with a high level of confidence, while the probability that the planet transits but that the eclipse was missed is nearly negligible $(0.26 \%)$.

To investigate the effect of the stellar size on this result, we performed a second MCMC analysis assuming $R_{*}=0.86 \pm$ $0.17 R_{\odot}$ instead of $0.68 \pm 0.02 R_{\odot}$ (see discussion in Sect. 2 ). The obtained transit probability was $0.63 \%$, leading to the conclusion that a transit of HD $40307 \mathrm{~b}$ can be considered as very unlikely, even if the star is actually significantly larger than deduced from its parallax and bolometric luminosity.
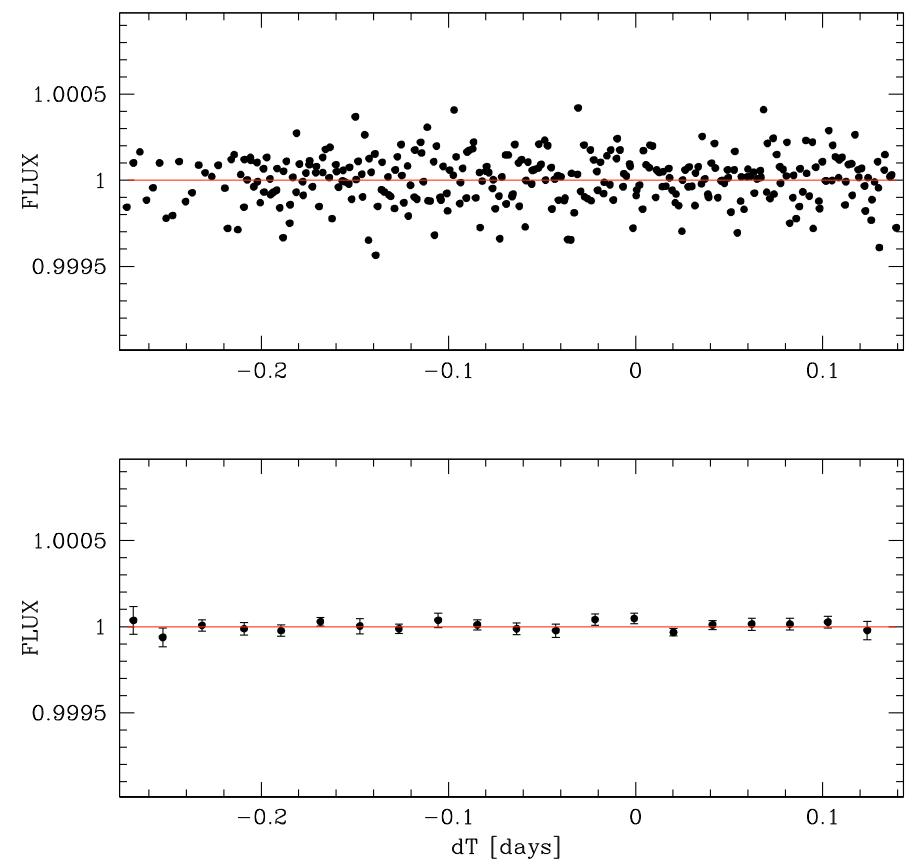

Fig. 6. Top: Light curve obtained after correction for the systematics of the three IRAC light curves used in this work and folding on the bestfitting ephemeris. Bottom: same but after binning with an interval of $30 \mathrm{~min}$.

Figure 6 shows the IRAC photometry corrected for the baselines of the three time series and period-folded on the best-fitting $T_{0}$. The rms of this light curve is $143 \mathrm{ppm}$ for a mean interval of $1.9 \mathrm{~min}$, while the rms goes down to $29 \mathrm{ppm}$ when the light curve is binned with an interval of $30 \mathrm{~min}$. This illustrates well the high photometric potential that the cryogenic Spitzer had at $8 \mu \mathrm{m}$. Now that its cryogen is depleted, only the 3.6 and $4.5 \mu \mathrm{m}$ channels remain operational, and similar photometric precisions are not guaranteed, mainly because of the inhomogeneous intrapixel sensitivity of the InSb detectors of IRAC (e.g. Knutson et al. 2008, and references therein). 


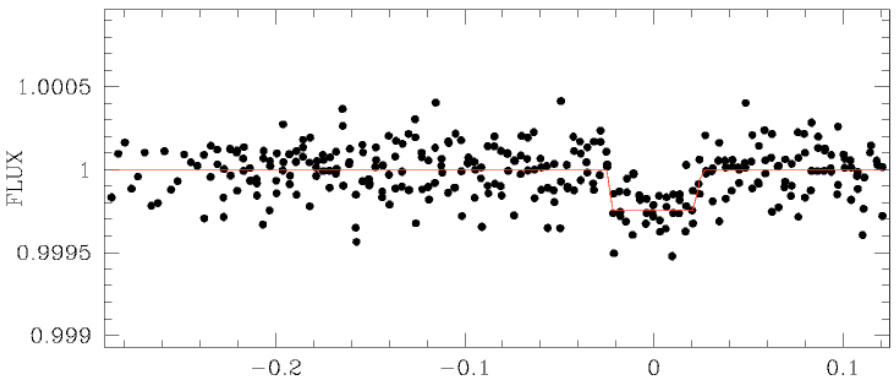

Fig. 7. Period-folded photometry with the best-fitting transit model obtained after insertion of a fake transit with a depth of $220 \mathrm{ppm}$ and a duration of $1 \mathrm{~h}$.

If present, the transit of a planet as small as $1.1 R_{\oplus}(220 \mathrm{ppm})$ should have been easily detected. To check this, we injected a fake transit of $\sim 1 \mathrm{~h}$ and $220 \mathrm{ppm}$ in the Spitzer photometry, using the values reported in Table 1 for $T_{0}$ and $P$. Our first MCMC analysis of these data was composed of two chains of $10^{6}$ steps. Except in the "burn-in" phases of the chains, a transit was present in all the steps of both chains, indicating a firm detection. To estimate a formal transit probability, we performed two new chains for which no transit was allowed. For both models (with and without transit), the marginal likelihood was computed from the MCMC samples following the description in Chib \& Jeliaskov (2001). The deduced Bayes ratio (e.g., Carlin \& Louis 2008) was $1.4 \times 10^{23}$ in favor of the model with a transit, confirming the very firm transit detection. Figure 7 shows the obtained best-fitting transit model superimposed on the periodfolded IRAC photometry corrected for the systematics. The deduced transit depth is $232 \pm 23 \mathrm{ppm}$, in good agreement with the actual value of $220 \mathrm{ppm}$.

We investigated the origin of the "transit" marginally detected in the first IRAC time series alone. It appears that it was most probably due to the baseline model used in our preliminary analysis. To recall, DD fitted a line in the light curve after cutting off the first three hours, while MG cut off the first hour and assumed a quadratic logarithmic function of time for the ramp model, i.e. the same function than shown in Eq. (1) but without the $a_{4} \mathrm{~d} t$ term. Figure 8 shows the photometry obtained after correction by the best-fitting baseline model obtained by least-square minimization, using Eq. (1) for the model, with and without the $a_{4} \mathrm{~d} t$ term. The residual correlated noise visible in the time series obtained when the $a_{4} \mathrm{~d} t$ term is not used could be easily "compensated" by a shallow transit, while the correlated noise is nearly negligible when the $a_{4} \mathrm{~d} t$ term is used. Using only the first IRAC light curve and the HARPS measurements as data, we performed two new MCMC analysis, one with and one without the $a_{4} \mathrm{~d} t$ term in the baseline equation. The stellar radius was set to $0.86 R_{\odot}$. Without the $a_{4} \mathrm{~d} t$ term, the deduced transit probability was $97.1 \%$, while it dropped to $0.9 \%$ with it. Comparing both models, the resulting Bayes ratio was 7.4 in favor of the model with the $a_{4} \mathrm{~d} t$ term. We conclude thus that we overestimated the significance of our transit detection in our preliminary analysis of the first IRAC light curve because we assumed too simple models for the baseline. This illustrates well how careful one should be when searching for very shallow transits in photometric time series presenting systematic effects of much larger amplitude, even if these systematics are rather well characterized.
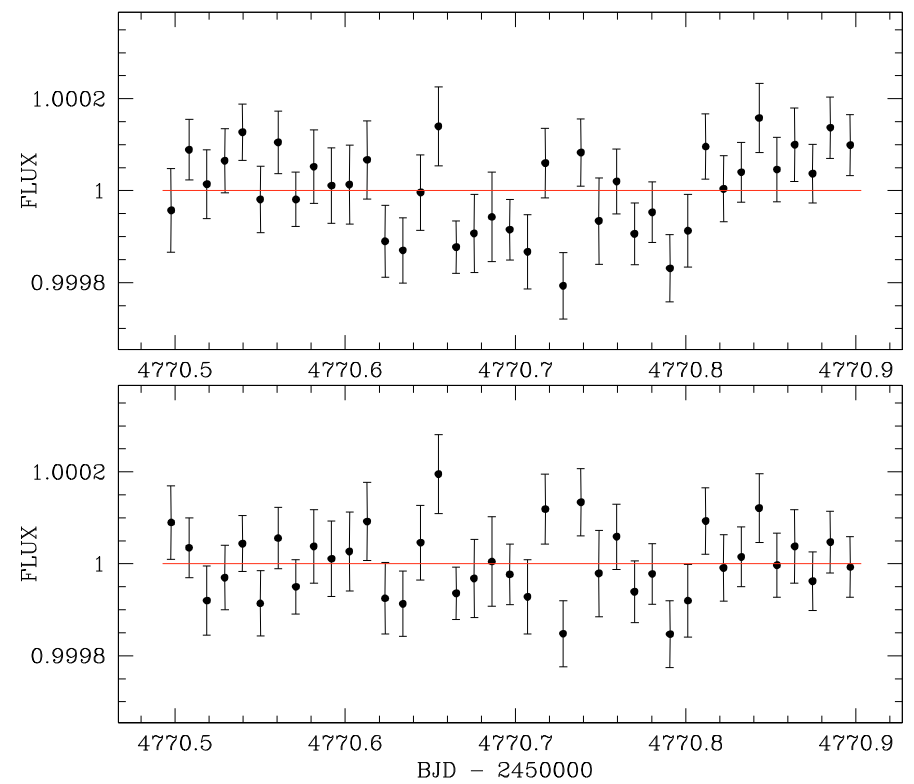

Fig. 8. Light curves for the first run corrected for the baseline model and binned with a five minutes interval, without (top) and with (bottom) the linear term $a_{4} \mathrm{~d} t$ in Eq. (1).

\section{Conclusions}

We have used Spitzer and its IRAC camera to search for the transit of the super-Earth HD 40307b. The transiting nature of the planet could not be firmly discarded from our first photometric monitoring of a transit window because of the uncertainty coming from the modeling of the photometric baseline. To obtain a firm result, two more transit windows were observed and a global Bayesian analysis of the three IRAC time series and the HARPS radial velocities was performed. Unfortunately, any transit of the planet during the observed phase window is firmly discarded, while the probability that the planet transits but that the eclipse was missed by our observations is nearly negligible $(0.26 \%)$.

Acknowledgements. This work is based in part on observations made with the Spitzer Space Telescope, which is operated by the Jet Propulsion Laboratory, California Institute of Technology under a contract with NASA. Support for this work was provided by NASA. M. Gillon acknowledges support from the Belgian Science Policy Office in the form of a Return Grant, and thanks J. Montalbán for her valuable suggestions about bolometric corrections.

\section{References}

Beaulieu, J.-P., Carey, S., Ribas, I., \& Tinetti, G. 2008, ApJ, 677, 1343

Butler, R. P., Vogt, S. S., Marcy, G. W., et al. 2004, ApJ, 617, 580

Bouchy, F., Udry, S., Mayor, M., et al. 2005, A\&A, 444, L15

Bouchy, F., Mayor, M., Lovis, C., et al. 2009, A\&A, 496, 527

Carlin, B. P., \& Louis, T. A. 2008, Bayesian Methods for Data Analysis, 3rd edn. (Chapman \& Hall/CRC)

Charbonneau, D., Brown, T. M., Latham, D. W., \& Mayor M. 2000, ApJ, 529, L45

Charbonneau, D., Brown, T. M., Noyes, R. W., \& Gilliland, R. L. 2002, ApJ, 568,377

Charbonneau, D., Knutson, H. A., Barman, T., et al. 2008, ApJ, 686, 1341

Charbonneau, D., Berta, Z. K., Irwin, J., et al. 2009, Nature, 462, 891

Chib, S., \& Jeliaskov, I. 2001, JASA, 96, 270

Deming, D. 2009, Transiting Planets, Proceedings of the International Astronomical Union, IAU Symp., 253, 197

Deming, D., Harrington, J., Laughlin, G., et al. 2007, ApJ, 667, L199

Demory, B.-O., Gillon, M., Barman, T., et al. 2007, A\&A, 475, 1125

ESA 1997, The HIPPARCOS and TYCHO catalogue, ESA-SP 1200

Forveille, T., Bonfils, X., Delfosse, X., et al. 2009, A\&A, 493, 645 
Gardner, J. P., Mather, J. C., Clampin, M., et al. 2006, Space Sci. Rev., 123, 485 Gelman, A., \& Rubin, D. 1992, Stat. Sci., 7, 457

Gillon, M., Demory, B.-O., Barman, T., et al. 2007a, A\&A, 471, L51

Gillon, M., Pont, F., Demory, B.-O., et al. 2007b, A\&A, 472, L13

Gillon, M., Smalley, B., Hebb, L., et al. 2009, A\&A, 496, 259

Gillon, M., Lanotte, A. A., Barman, T., et al. 2010, A\&A, 511, A3

Giménez, A. 2006, ApJ, 650, 408

Henry, G. W., Marcy, G. W., Butler, R. P., \& Vogt, S. S. 2000, APJ, 529, L4

Knutson, H. A., Charbonneau, D., Allen, L. E., et al. 2007, Nature, 447, 183

Knutson, H. A., Charbonneau, D., Allen, L. E., et al. 2008, ApJ, 673, 526

Johnson, J. A., Winn, J. N., Cabrera, N. E., \& Carter, J. A. 2009, ApJ, 692, L100

Léger, A., Rouan, D., Schneider, J., et al. 2009, A\&A, 506, L287

Lovis, C., Mayor, M., Bouchy, F., et al. 2009, Transiting Planets, Proceedings of the International Astronomical Union, IAU Symp., 253, 502

Massana, E., Jordi, C., \& Ribas, I. 2006, A\&A, 450, 735

Mandel, K., \& Agol, E. 2002, ApJ, 580, 171

Mayor, M., Pepe, F., Queloz, D., et al. 2003, The Messenger, 114, 20

Mayor, M., Udry, S., Lovis, C., et al. 2009a, A\&A, 493, 639
Mayor, M., Bonfils, X., Forveille, T., et al. 2009b, A\&A, 507, 487 Queloz, D., Bouchy, F., Moutou, C., et al. 2009, A\&A, 506, 303 Rogers, L. A., \& Seager, S. 2010, ApJ, 716, 1208

Santos, N. C., Bouchy, F., Mayor, M., et al. 2004, A\&A, 426, L19

Seager, S., Kuchner, M., Hier-Majumder, C. A., \& Militzer B. 2007, ApJ, 669, 1279

Seager, S., Deming, D., \& Valenti, J. A. 2009, Astrophys. Space Sci. Proc., ISBN 978-1-4020-9456-9. (Netherlands: Springer), 123

Sousa, S., Santos, N. C., Mayor, M., et al. 2008, A\&A, 487, 373

Skrutskie, M. F., Cutri, R. M., Stiening, R., et al. 2006, AJ, 131, 1163

Swain, M. E., Vasisht, G., \& Tinetti, G. 2008, Nature, 452, 329

Torres, G., Andersen, J., \& Giménez, A. 2009, A\&AR, 13

Udry, S., Bonfils, X., Delfosse, X., et al. 2007, A\&A, 469, 43

Valencia, D., Masahiro, I., Guillot, T., \& Nettelman, N. 2010, A\&A, 516, A20

Vidal-Majar, A., Lecavelier des Etangs, A., Désert, J.-M., et al. 2003, Nature, 422,143

Werner, M. W., Roellig, T. L., Low, F. J., et al. 2004, ApJS, 154, 1

Winn, J. N., Holman, M. J., Carter, J. A., et al. 2009, AJ, 137, 3826 